cond-mat/0210630

\title{
Hard-sphere limit of soft-sphere model for granular materials: Stiffness dependence of steady granular flow
}

\author{
Namiko Mitara:* and Hiizu Nakanish \\ Department of Physics, Kyushu University 33, Fukuoka 812-8581, Japan
}

(Dated: February 1, 2008)

\begin{abstract}
Dynamical behavior of steady granular flow is investigated numerically in the inelastic hard sphere limit of the soft sphere model. We find distinctively different limiting behaviors for the two flow regimes, i.e., the collisional flow and the frictional flow. In the collisional flow, the hard sphere limit is straightforward; the number of collisions per particle per unit time converges to a finite value and the total contact time fraction with other particles goes to zero. For the frictional flow, however, we demonstrate that the collision rate diverges as the power of the particle stiffness so that the time fraction of the multiple contacts remains finite even in the hard sphere limit although the contact time fraction for the binary collisions tends to zero.
\end{abstract}

PACS numbers: $45.70 . \mathrm{Mg}, 45.50 .-\mathrm{j}$

*Electronic address: namiko@stat.phys.kyushu-u.ac.jp

$\dagger$ Electronic address: naka4scp@mbox.nc.kyushu-u.ac.jp 


\section{INTRODUCTION}

The interactions between grains in flowing granular material are roughly classified into two types; the impulsive contact (collision) with the momentum exchange and the sustained contact with the transmission of forces [1]. The flow in which the impulsive contact is dominant is called collisional flow, while the flow where the sustained contact dominates is called frictional flow. These two types of flow may be found in a simple geometry such as granular flow on a slope. The grains stay at rest when the inclination angle is too small. If the inclination exceeds a critical angle, the material starts flowing frictionally at first. The flow becomes collisional when the inclination is large enough.

As for the collisional flow of granular material, its dynamics has some analogy with molecular fluid, and the kinetic theories based on inelastic binary collisions of particles hold to some extent 2]. On the other hand, the frictional flow is drastically different from the molecular fluid, and we have little understanding on it. Many models have been proposed for dense granular flows: For example, some models take into account the effect of non-

local force transmission which comes from the network of contacting grains [3]. In the experiments, however, it is difficult to specify the nature of sustained contact in the dense flow.

For the simulations of granular dynamics, the following two models have been commonly used, i.e. the inelastic hard sphere model and the soft sphere model.

In the inelastic hard sphere model, the particles are rigid and the collisions are thus instantaneous, therefore its dynamics can be defined through a few parameters that characterize a binary collision because there are no many-body collisions [4]. The model is simple and there are very efficient algorithms to simulate it [5], but it describes basically only the collisional flow [6] because the sustained contact is not allowed. It is also known that the system often encounters what is called the inelastic collapse [7, 8, 9, 10]; the infinite number of collisions take place among a small number of particles in a finite time, thus the dynamics cannot be continued beyond that point without additional assumption.

On the other hand, in the soft sphere model, that is sometimes called the discrete element method (DEM) in the granular community [4, 11], the particles overlap during collision and the dynamics is defined through the forces acting on the colliding particles. Collision takes finite time, and not only binary collision but also many-body collision and sustained contact 
between particles are possible, therefore, both the collisional and the frictional flows may realize in the model. Many researches have been done on granular flow down a slope using the DEM in both the collisional regime and the frictional regime [12, 13, 14].

In actual simulations, however, the stiffness constants used in the soft sphere model are usually much smaller than the one appropriate for real material such as steel or glass ball 15] because of numerical difficulty. Therefore, some part of sustained contact in simulations may be decomposed into binary collisions if stiffer particles are used. It is also not clear that the frictional force in the sustained contact may be described by the same forces with the one suitable for the collisional events.

It is, therefore, important to examine how the system behavior may change as the stiffness constant increases in the soft sphere model, or in other words, how the soft sphere model converges to the inelastic hard sphere model in the infinite stiffness limit. In this paper, we present the results of numerical simulations of the granular flow using the soft sphere model, and investigate the system behavior when we change the stiffness constant systematically with keeping the resulting restitution constant unchanged.

In Sec. II, we briefly summarize an inelastic hard sphere model that is used for collisional granular flows. Then we introduce a simple soft sphere model for granular material and discuss how we take the hard sphere limit keeping the restitution coefficient constant. In Sec. III the simulation results are shown. At first, the stiffness dependence of the steady state of a single particle rolling down a slope is presented to see how the inelastic collapse appears in the hard sphere limit. The collisional flow and the frictional flow are examined. We find that the interactions between particles in the collisional flow smoothly converge to binary collisions of inelastic hard spheres, while those in frictional flow shows non-trivial behavior; the behavior is also different from the "inelastic collapse" in the single particle system. The summary and the discussion are given in Sec. IV.

\section{HARD SPHERE MODEL AND SOFT SPHERE MODEL}

In this section, we introduce the hard sphere model and the soft sphere model for granular material. For simplicity, we consider a two dimensional system and grains are modeled by two dimensional disks.

After showing the correspondence of parameters in the soft sphere model to those of the 
hard sphere model, we briefly summarize the phenomenon called inelastic collapse, which is the singular behavior in the inelastic hard sphere system. At the last part of this section, we discuss the limiting behavior of the soft sphere model that corresponds to the inelastic collapse of the hard sphere model.

\section{A. Inelastic hard sphere model}

In hard sphere models, collisions between particles or between a particle and a wall are considered to happen instantaneously, and its dynamics is defined by the binary collision rule. We consider the collision rule in terms of the normal and tangential restitution coefficients and the sliding friction [6, 16].

Let us consider a collision between the two spheres $i$ and $j$ of the diameters $\sigma_{i}$ and $\sigma_{j}$ and the masses $m_{i}$ and $m_{j}$ at the contact positions $\boldsymbol{r}_{i}$ and $\boldsymbol{r}_{j}$, respectively. Prior to the collision, the disks have velocities $\boldsymbol{c}_{i}$ and $\boldsymbol{c}_{j}$ and angular velocities $\boldsymbol{\omega}_{i}$ and $\boldsymbol{\omega}_{j}$. Then the relative velocity of the point of contact $\boldsymbol{v}_{i j}$ is given by

$$
\boldsymbol{v}_{i j}=\left(\boldsymbol{c}_{i}-\boldsymbol{c}_{j}\right)+\boldsymbol{n} \times\left(\frac{\sigma_{i}}{2} \boldsymbol{\omega}_{i}+\frac{\sigma_{j}}{2} \boldsymbol{\omega}_{j}\right),
$$

where the normal vector $\boldsymbol{n}=\boldsymbol{r}_{i j} /\left|\boldsymbol{r}_{i j}\right|=\left(n_{x}, n_{y}, 0\right)$ with $\boldsymbol{r}_{i j}=\boldsymbol{r}_{i}-\boldsymbol{r}_{j}$.

If $\boldsymbol{v}_{i j}^{\prime}$ denotes the post-collisional relative velocity, the collision rule for normal direction is

$$
\left(\boldsymbol{n} \cdot \boldsymbol{v}_{i j}^{\prime}\right)=-e\left(\boldsymbol{n} \cdot \boldsymbol{v}_{i j}\right)
$$

where $e$ is the normal restitution coefficient with $0 \leq e \leq 1$.

In the case without sliding, the collision rule in the tangential direction is given by

$$
\left(\boldsymbol{n} \times \boldsymbol{v}_{i j}^{\prime}\right)=-\beta\left(\boldsymbol{n} \times \boldsymbol{v}_{i j}\right),
$$

where $\beta$ is the tangential restitution coefficient with $-1 \leq \beta \leq 1$. The sliding is taken into account so that the tangential component of impulse does not exceed $\mu|\boldsymbol{n} \cdot \boldsymbol{J}|$ with the Coulomb friction coefficient $\mu$, where $\boldsymbol{J}$ is the momentum change of the particle $i$ through the collision. Namely, when the momentum change of the particle $i$ through the collision rules (2) and (3) is $\boldsymbol{J}^{\text {nos }}$, then $\left(\boldsymbol{n} \times \boldsymbol{v}_{i j}^{\prime}\right)$ is determined so that

$$
|\boldsymbol{n} \times \boldsymbol{J}|=\min \left(\mu|\boldsymbol{n} \cdot \boldsymbol{J}|,\left|\boldsymbol{n} \times \boldsymbol{J}^{\text {nos }}\right|\right)
$$

is satisfied. The detailed description of the rule is given in Refs. 6, 16]. 


\section{B. Soft sphere model: DEM}

The DEM, or the soft sphere model [4, 11], is often used to simulate the dynamics of granular materials. In the present work, we adopt the two dimensional one with the linear elastic force and dissipation. When the two disks $i$ and $j$ at positions $\boldsymbol{r}_{i}$ and $\boldsymbol{r}_{j}$ with velocities $\boldsymbol{c}_{i}$ and $\boldsymbol{c}_{j}$ and angular velocities $\boldsymbol{\omega}_{i}$ and $\boldsymbol{\omega}_{j}$ are in contact, the force acting on the particle $i$ from the particle $j$ is calculated as follows: The normal velocity $v_{n}$, the tangential velocity $v_{t}$, and the tangential displacement $u_{t}$ are given by

$$
\begin{array}{r}
v_{n}=\boldsymbol{n} \cdot \boldsymbol{v}_{i j}, \quad v_{t}=\boldsymbol{t} \cdot \boldsymbol{v}_{i j}, \\
u_{t}=\int_{t_{0}}^{t} v_{t} \mathrm{~d} t
\end{array}
$$

where $\boldsymbol{v}_{i j}$ is given in Eq. (11), and $\boldsymbol{t}=\left(-n_{y}, n_{x}, 0\right)$. Here, $t_{0}$ is the time when the two particles

start to be in contact. Then the normal force $F_{i j}^{n}$ and the tangential force $F_{i j}^{t}$ acting on the particle $i$ from the particle $j$ are given by

$$
\begin{aligned}
F_{i j}^{n} & =2 M k_{n}\left(\frac{\sigma_{i}+\sigma_{j}}{2}-\left|\boldsymbol{r}_{i j}\right|\right)-2 M \eta_{n} v_{n}, \\
F_{i j}^{t} & =\min \left(\left|h_{t}\right|, \mu\left|F_{n}\right|\right) \operatorname{sign}\left(h_{t}\right)
\end{aligned}
$$

with

$$
h_{t}=-2 M k_{t} u_{t}-2 M \eta_{t} v_{t},
$$

where $k_{n}$ and $k_{t}$ are the elastic constants, $\eta_{n}$ and $\eta_{t}$ are the damping parameters, $\mu$ is the Coulomb friction coefficient for sliding, and $M=m_{i} m_{j} /\left(m_{i}+m_{j}\right)$ is the reduced mass.

\section{Hard sphere limit of soft sphere model}

When we adopt the linear dependence of the elastic and viscous force on the overlap and the normal relative velocity as in Eq. (7), we can calculate the duration of contact $\tau_{c}$ and the restitution coefficient for a normal binary collision $e$ [17]; they are given by

$$
\tau_{c}=\frac{\pi}{\sqrt{2 k_{n}-\eta_{n}^{2}}}
$$

and

$$
e=\exp \left(-\frac{\pi \eta_{n}}{\sqrt{2 k_{n}-\eta_{n}^{2}}}\right)
$$


respectively.

Neglecting the sliding friction and the variation of $\boldsymbol{n}$ during the contact, the half period of the oscillation in the tangential displacement $u_{t}$ is estimated as [17]

$$
\tau_{s}=\frac{\pi}{\sqrt{6 k_{t}-9 \eta_{t}^{2}}}
$$

for the two dimensional disks with the moment of inertia $I_{i}=m_{i} \sigma_{i}^{2} / 8$. Here we choose the parameters $k_{t}$ and $\eta_{t}$ so that the relation $\tau_{s}=\tau_{c}$, or,

$$
\sqrt{6 k_{t}-9 \eta_{t}^{2}}=\sqrt{2 k_{n}-\eta_{n}^{2}}
$$

is satisfied. Under this condition, the tangential restitution coefficient is given by

$$
\beta=\exp \left(-3 \eta_{t} \tau_{c}\right)
$$

Equations (10) and (11) can be rewritten as

$$
\begin{aligned}
\eta_{n} & =\left[\frac{2 k_{n}}{(\pi / \ln e)^{2}+1}\right]^{1 / 2} \\
\tau_{c} & =\left[\frac{\pi^{2}+(\ln e)^{2}}{2 k_{n}}\right]^{1 / 2}
\end{aligned}
$$

Using Eqs. (13)-(16), we can take the inelastic hard sphere limit of this model for given $e$ and $\beta$ by taking the $k_{n} \rightarrow \infty$ limit; $\eta_{n}, \eta_{t}$, and $k_{t}$ are given by Eqs. (15), (14), and (13), and the duration time of collision $\tau_{c}$ goes to zero as Eq. (16).

\section{Inelastic collapse}

It is well known that the inelastic hard sphere system can undergo the inelastic collapse, i.e., the phenomenon where infinite collisions take place within a finite period of time [7]. The simplest example is the vertical bouncing motion of a ball under gravity, but it has been shown that the inelastic collapse also occurs in higher dimensional systems without gravity [7, 8, 9, 10].

The inelastic collapse never occurs in the soft sphere model because of the finite length of the collision time. However, it is worth to discuss what will happen in the soft sphere model in the simple situation where the inelastic collapse occurs in the hard sphere model. First, let us consider the vertical bouncing motion of a soft ball under gravity. We assume 
the same force law between the ball and the floor as in Eqs. (7) and (8), except that we replace $2 M$ by the mass of the ball. While the ball and the floor are in contact, the equation of motion for the overlap of the ball and the floor, $z$, is given by

$$
\ddot{z}+k_{n} z+\eta_{n} \dot{z}=g
$$

with the acceleration of gravity $g$, where the dots represent the time derivatives. One can show that, if the impact velocity $v_{i}$ is below a critical value $v_{c}$, which is $O\left(1 / \sqrt{k_{n}}\right)$ for large enough $k_{n}$, the ball stays in contact with the floor; for $v_{i} \gg v_{c}$, the restitution coefficient $e_{w}$ can be considered as a constant. Therefore, for a given initial impact velocity $v_{0}$, the number of necessary collisions $n_{c}$ for the ball to stay in contact with the floor is roughly estimated by the condition $e_{w}^{n_{c}} v_{0} \sim v_{c}$, namely, $n_{c} \sim \ln \left(v_{c} / v_{0}\right) / \ln e_{w}$. Because $v_{c} \sim 1 / \sqrt{k_{n}}$ for large $k_{n}, n_{c}$ behaves as

$$
n_{c} \propto \ln k_{n}+\text { const. }
$$

in the hard sphere limit. Thus $n_{c}$ diverges logarithmically when $k_{n} \rightarrow \infty$, which corresponds to the inelastic collapse due to gravity.

In the case without gravity, the inelastic collapse results in the "many-body collision" in the soft sphere model. For example, we consider three soft spheres in one dimension and assume that the binary collision can be approximated by the collision law with a constant restitution coefficient. In the situation where a particle goes back and forth between the two particles approaching each other, the inelastic collapse may take place in the hard sphere model [8]; three balls lose relative velocity completely in the limit of infinite collisions. In the soft sphere model, however, when the interval between two collisions becomes smaller than the duration of contact $\tau_{c}$, three balls are in contact at the same time, namely, the three body collision occurs; then they will fly apart. The number of collisions before the three body collision will also diverge logarithmically in the hard sphere limit because $\tau_{c} \propto 1 / \sqrt{k_{n}}$.

On the other hand, one should note that a many-body collision in the soft sphere model does not necessarily result in the inelastic collapse in the hard sphere limit. Actually, in most of the cases, a many-body collision will be decomposed into a set of binary collisions in the hard sphere limit. 


\section{SIMULATION RESULTS}

In this section, we investigate the stiffness dependence of granular material on a slope in the following three situations: (i) a single particle rolling down a slope, (ii) the dilute collisional flow, and (iii) the dense frictional flow. We focus on the steady state in each situation and compare the simulation data with changing $k_{n}$ systematically. The particles are monodisperse in (ii), while they are polydisperse in (iii) in order to avoid crystallization.

In the simulations, the parameters have been chosen to give $e=\beta=0.7, \mu=0.5$ in the hard sphere limit. All values are non-dimensionalized by the length unit $\sigma$, the mass unit $m$,

and the time unit $\sqrt{\sigma / g}$. Here, $\sigma$ is the diameter of the largest particle in the system and $m$ is the mass of that particle. The second order Adams-Bashforth method and the trapezoidal rule are used to integrate the equations for the velocity and the position, respectively [18]. Note that the time step for integration, $d t$, needs to be adjusted as $\tau_{c}$ becomes smaller. All the data presented in the paper are results with $d t=\min \left(\tau_{c} / 100,10^{-4}\right)$. We have confirmed that the results do not change for $d t \leq \tau_{c} / 100$ by calculating also with $d t=\tau_{c} / 50$ and $d t=\tau_{c} / 200$ in the case of the single particle.

\section{A. A single particle rolling down a bumpy slope}

Let us first consider a single particle rolling down a bumpy slope. It is known that the particle shows the steady motion for a certain range of the inclination angle $\theta[19]$. In the simulations, we make the boundary rough by attaching the same particles with the rolling one to the slope with the spacing $0.002 \sigma$ (see Fig. 11). For the chosen parameters with the normal stiffness $k_{n}=2^{-1} \times 10^{5}$, the range of $\theta$ for which steady motion is realized is $0.11 \lesssim \sin \theta \lesssim 0.14$ [13]. Here we fix the inclination angle to $\sin \theta=0.13$. Figure 2 shows the time evolution of the velocity in the $y$ direction, $v_{y}$, with $k_{n}=2^{-1} \times 10^{5}$ (solid line) and $k_{n}=2^{17} \times 10^{5}$ (dashed line). It is shown that $v_{y}$ behaves periodically; this period $(\Delta t \sim 4)$ corresponds to the period for the particle to get past one particle at the floor. The period hardly depends on the stiffness.

In Fig. 3 (a), the $k_{n}$ dependence of the time averaged kinetic energy $E$ is shown. It is shown that the energy is an increasing function of $1 / k_{n}$ in the softer region $\left(1 / k_{n} \gtrsim 10^{-7}\right)$, but no systematic $1 / k_{n}$ dependence of $E$ exists for $1 / k_{n} \lesssim 10^{-7}$. The average collision 
rate (number of collisions per unit time) between the slope and the particle, $N_{w}$, shows logarithmic dependence on $1 / k_{n}$ in the whole region (Fig. 3 (b)). From Fig. 3 (c), we also find that the average contact time fraction with the slope, $t_{w}$, is a decreasing function of $k_{n}$ in the soft region, but it seems to approach a constant value for large enough $k_{n}$.

The logarithmic $k_{n}$ dependences of $N_{w}$ and the constant $t_{w}$ in large $k_{n}$ region agree with our previous analysis of "inelastic collapse under gravity" in the soft sphere model in Section IID. The motion of the particle in one period is as follows; when the particle comes to a bump (a particle attached to the slope), the particle jumps up, bounces on the bump many times, loses the relative velocity, and finally rolls down keeping in contact with the bump. Therefore, the contact time fraction has a finite value even in the hard sphere limit due to the rolling motion at the last part. $N_{w}$ increases logarithmically in the hard sphere limit as has been expected from Eq. (18).

\section{B. Collisional flow}

Next we consider the steady state of the collisional flow. The system considered is shown in Fig. 4. The particles are monodisperse, and the slope is made rough as in the single particle case. The periodic boundary condition is adopted in the flow direction ( $x$ direction). The length of the slope is $L=50.1$ and the number of the particles attached to the slope is 50. The number of flowing particles is also 50, namely, the number of the particles per unit length along the slope is about 1 . The inclination angle is set to be $\sin \theta=0.45$. The initial configuration of particles is the row of 50 particles at rest with regular spacing in the $x$-direction, but each particle is at random height in the $y$-direction. After a short initial transient, if the total kinetic energy fluctuates around a certain value, we consider it as the steady flow. All the data were taken in this regime and averaged over the time period of 1500 .

As can be seen in the snapshot, Fig. 4, the particles bounce and the number of particles in contact is very small. In Fig. 5 (a), the averaged kinetic energy per one particle, $E$, is shown. Though $E$ becomes larger as the particles become softer in $1 / k_{n} \gtrsim 10^{-5}$, the

systematic dependence of $E$ on $k_{n}$ disappears for large enough $k_{n}\left(1 / k_{n} \lesssim 10^{-6}\right)$ [20]. The $y$-dependence of the average number density and the flow speed in this region are also shown in Fig. 6 (a) and (b). 
In Fig. [5 (b), the average collision rates between particles, $N_{c}$ (filled circles), and between particles and the slope, $N_{w}$ (open circles), per particle are plotted vs $1 / k_{n}$. Both of them stay roughly constant in the region where $E$ is almost constant, $1 / k_{n} \lesssim 10^{-6}$. On the other hand, the average time fractions during which a particle is in contact with other particles, $t_{c}$ (filled circles), and in contact with the slope, $t_{w}$ (open circles), decrease systematically as $k_{n}$ increases as shown in Fig. 5(c). Both the solid and the dashed lines are proportional to $1 / \sqrt{k_{n}}$, namely, $t_{c}$ decreases in the same manner as $\tau_{c}$ in Eq. (16). Actually, the collision time fractions $t_{c}$ and $t_{w}$ converge to $N_{c} \tau_{c}$ and $N_{w} \tau_{w}\left(\tau_{w}\right.$ is the duration of a normal collision of a particle and the floor [21] ), respectively, in the hard sphere limit. The differences $t_{c}-N_{c} \tau_{c}$ and $t_{w}-N_{w} \tau_{w}$ are plotted in Fig. [5] (d) to show that they go to zero very rapidly upon increasing $k_{n}$. This means that the interactions of the soft sphere model in the collisional flow regime converge to those of the inelastic hard sphere model with binary collisions.

If we look carefully, however, in the large $k_{n}$ region in Fig. [5](c), we can see slight deviation of $t_{w}$ from the dashed lines; it decreases slower than $1 / \sqrt{k_{n}}$. This tendency may indicate that $t_{w}$ remains finite in the hard sphere limit: Actually, in the event-driven simulation of the hard sphere model, we always found the inelastic collapse as long as the restitution constant between a particle and the floor is less than 1 . This suggests that $N_{w}$ should diverge and $t_{w}$ should remain finite in the hard sphere limit because of the inelastic collapse due to gravity. The slight deviation of $t_{w}$ from the dashed line in Fig. [5 (c) may be a symptom of it, while we cannot see the logarithmic divergence in $N_{w}$.

\section{Frictional flow}

Now we study the steady state of the dense frictional flow. We adopted the flat boundary with slope length $L=10.02$ and imposed the periodic boundary condition in the flow direction. In order to avoid crystallization, we used the polydisperse particles with the uniform distribution of diameter from 0.8 to 1.0. The number of particles in the system is 100. The inclination angle $\theta$ is set to be $\sin \theta=0.20$ and the initial condition is given in

the similar way with the collisional flow. The ten rows of ten particles with regular spacing in the $x$-direction are at rest with large enough spacings between rows in the $y$-direction so that particles do not overlap; only the particles in the top row are at random heights in $y$-direction. 
It turns out that the steady state is not unique and fluctuation is large. Depending on the initial conditions, the particles may flow with a different value of average kinetic energy, or in some cases, the whole system stops. We presume that this is mainly because the system is not large enough: Grains form a layer structure as in Fig. 7, and the flow velocity strongly depends on the configuration of particles in the bottom layer. The time evolutions of the kinetic energy per particle, $E(t)$, of three samples with different configurations in the bottom layer are shown in Fig 8 . Sample 1 shows stable behavior while sample 2 eventually stops after running with lower energy for some time. Sample 3 shows larger fluctuation with higher energy; the sudden change of $E(t)$ in sample 3 results from the change in the configuration of the bottom layer. This large fluctuation should be averaged out if we could simulate large enough system for long enough time, but the amount of computation is too large especially for the system with stiff particles.

In order to make meaningful comparison out of these largely fluctuating data with a variety of behaviors, we select simulation sequences that come from similar flowing behaviors in the following way. First, we define the steady part of the time sequence in each of the samples as the part where the width of the energy fluctuations is smaller than 0.45 over the time period longer than 500. Second, we exclude the data whose averaged energy is out of the range $[0.4,0.8]$ [22].

Many of the excluded data by this criterion show quite different flowing behaviors. We use only the data selected from this criterion to calculate time averages of physical quantities.

In the snapshot of the frictional flow, Fig. 7] most of the particles seem to be in contact with each other and form a layer structure. The $y$-dependence of the average density and the flow speed are shown in Figs. 9 (a) and (b), respectively. We can see that the relative motion between layers is largest at the bottom and very small in the bulk. The stiffness dependence of the average kinetic energy $E$ is shown in Fig. 10 (a); the data are scattered due to the non-uniqueness of the steady state.

Nevertheless, the average collision rates show systematic dependence as shown in Fig. 10 (b). Here, the definition of $N_{c}\left(t_{c}\right)$ is the same as that for the collisional flow, namely, it is the average collision rate (contact time fraction) between particles per particle in the system. On the other hand, $N_{w}\left(t_{w}\right)$ is defined differently; it is the collision rate (the contact time fraction) between the particles and the slope per particle in the bottom layer, because other particles never touch the slope. 
In Fig. 10 (b), we can see that $N_{c}$ (filled circles) and $N_{w}$ (open circles) increase very rapidly as $k_{n}$ becomes larger: they diverge as a power of $k_{n}$. This is quite different from the behavior in the collisional flow in which they are almost constant. Furthermore, this increase is faster than the logarithmic divergence found in the single particle case (see Fig. [3 (b)).

The contact time fractions, $t_{c}$ (filled circles) and $t_{w}$ (open circles), decrease as shown in Fig. 10 (c). The main reason why $t_{c}$ and $t_{w}$ decrease is that $\tau_{c}$ and $\tau_{w}$ decrease faster than $N_{c}$ and $N_{w}$, namely, the contact time fractions estimated from the duration of a binary collision, $N_{c} \tau_{c}$ and $N_{w} \tau_{w}$, continue to decrease. Actually, the decrease of the contact time fraction and the increase of the collision rate are natural because a longer multiple contact breaks up into shorter binary contacts as the particles become stiffer.

These contact time fractions, $t_{c}$ and $t_{w}$, however, do not converge to $N_{c} \tau_{c}$ and $N_{w} \tau_{w}$, respectively. As shown in Fig. [10 (d), $t_{c}-N_{c} \tau_{c}$ (filled circles) and $t_{w}-N_{w} \tau_{w}$ (open circles) are very large as compared to those in the collisional flow, even in the stiffest region. The comparison of this with the rapid convergence in the collisional flow regime (Fig. 5 (d)) indicates that there remains finite multiple contact time in the hard sphere limit and the interaction in the frictional flow can never be considered as many, or even infinite, instantaneous binary collisions. The particles experience the lasting multiple contact even in the hard sphere limit.

\section{SUMMARY AND DISCUSSION}

The inelastic hard sphere limit of granular flow has been investigated numerically in the steady states of (i) a single particle rolling down the slope, (ii) the dilute collisional flow, and (iii) the dense frictional flow. In (i), it has been found that the "inelastic collapse" between

the particle and the slope occurs in the hard sphere limit due to gravity, and the contact time fraction between the particle and the slope remains finite. In (ii), the collision rates $N_{c}$ and $N_{w}$ are almost constant when particles are stiff enough. The contact time fraction between particles $t_{c}$ approaches zero as $k_{n}$ increase in the same manner with the duration of contact for binary collision, $\tau_{c}$. This means that the interaction between particles in the hard sphere limit can be expressed by binary collisions in the inelastic hard sphere model. On the other hand, the decrease in $t_{w}$ is slightly slower than $1 / \sqrt{k_{n}}$ in the harder region, 
which can be a sign of the inelastic collapse between a particle and the slope.

In the case of the frictional flow (iii), the situation is not simple: Although the contact time fractions $t_{c}$ and $t_{w}$ decrease upon increasing $k_{n}$, the collision rates $N_{c}$ and $N_{w}$ increase as a power of $k_{n}$, which is faster than the logarithmic divergence found in the single particle case (i). The origin of this power divergence of collision rate does not seem to be simple because it is a property of the steady state, not a particular dynamical trajectory of the system.

The multiple contact time fraction may be estimated by $t_{c}-N_{c} \tau_{c}$ and $t_{w}-N_{w} \tau_{w}$. They were found to be quite large compared to those for the collisional flow, and seems to remain finite even in the infinite stiffness limit: This suggests that the interaction in the frictional flow can never be considered as infinite number of binary collisions. Even in the hard sphere limit, particles experience the lasting multiple contact.

The non-negligible fraction of multiple contact in the hard sphere limit implies the existence of the network of contacting grains even though they are flowing. The models of dense flows should consider the effect of this lasting contacts.

Here we have investigated only the two cases, i.e., the collisional flow and the frictional flow. The system should undergo the transition between the two flows if we change continuously the parameters such as the inclination angle, the roughness of the slope, or the density of particles. It is interesting to investigate how the transition occurs by looking at the quantities measured in this paper, because their stiffness dependences are qualitatively different in the two flows.

\section{Acknowledgments}

This research was partially supported by Hosokawa powder technology foundation, the Japan Society for the Promotion of Science, and Grant-in-Aid from the Ministry of Education, Science, Sports and Culture of Japan.

[1] S. B. Savage, Adv. Appl. Mech. 24, 289 (1984); C. Ancey, Phys. Rev. E 65, 011304 (2002).

[2] J. T. Jenkins and S. B. Savage, J. Fluid Mech. 130, 187 (1983); C. S. Campbel, Annu. Rev. Fluid Mech. 22, 57 (1990); I. Goldhirsch, in Modeling in Applied Sciences: A Kinetic 
Theory Approach, ed. N. Bellomo and M. Pulvirenti (Birkhauser, Boston, 2000); N. Mitarai, H. Hayakawa, and H. Nakanishi, Phys. Rev. Lett. 88, 174301 (2002).

[3] P. Mills, D. Loggia, and M. Tixier, Europhys. Lett. 45, 733 (1999); B. Andreotti and S. Douady, Phys. Rev. E 63, 031305 (2001).

[4] J. Duran, Sands, Powders, and grains: an introduction to the physics of granular materials (Springer, New York, 1997).

[5] M. Isobe, Int. J. Mod. Phys. C10, 1281 (1999).

[6] M. Y. Louge, Phys. Fluids 6, 2253 (1994).

[7] L. P. Kadanoff, Rev. Mod. Phys. 71, 435 (1999).

[8] B. Berne and R. Mazighi, J. Phys. A: Math. Gen. 23, 5745 (1990).

[9] S. McNamara and W. R. Young, Phys. Fluids A 4, 496 (1992).

[10] S. McNamara and W. R. Young, Phys. Rev. E 50, R28 (1994); 53, 5089 (1996); N. Schorghofer and T. Zhou, ibid. 54, 5511 (1996); M. Alam and C. M. Hrenya, ibid. 63, 061308 (2001).

[11] P. A. Cundall and O. D. L. Strack, Geotechnique 29, 47 (1979); A. Shimosaka, in Funtai Simulation Nyumon, edited by The Society of Powder Technology, Japan (Sangyo Tosho, Tokyo, 1998) p. 34, Sec. 3.2.

[12] T. Pöschel, J. Phys. II (France) 3, 27 (1993).

[13] N. Mitarai and H. Nakanishi, J. Phys. Soc. Jpn. 70, 2809 (2001).

[14] D. Ertas et al., Europhys. Lett. 56, 214 (2001); L. E. Silbert, G. S. Grest, S. J. Plimpton, and D. Levine, Phys. Fluids 14, 2637 (2002); L. E. Silbert et al., Phys. Rev. E 64, 051302 (2002).

[15] S. Yuu, T. Abe, T. Saitoh, and T. Umekage, Adv. Powder Technol. 6, 259 (1995).

[16] S. Luding, Phys. Rev. E 52, 4442 (1995).

[17] J. Schafer, S. Dippel, and D. E. Wolf, J. Phys. I (France) 6, 5 (1996).

[18] P. Henrici, Discrete Variable Methods in Ordinary Differential Equations (Wiley, New York, 1962).

[19] S. Dippel, G. G. Batrouni, and D. E. Wolf, Phys. Rev. E 54, 6845 (1996).

[20] The stiffness dependence of the motion of particles discharging from a hopper using discrete element method has been studied by Yuu et al [15]. They performed the simulations with three different values of stiffness and found the different behaviors. The comparison with the present results is not simple because of the different set up, but if we normalize their parameter of normal stiffness with the particle mass, the diameter, and the acceleration of gravity, then 
their values extend over the range where $E$ depends on $k_{n}$ in our simulations.

[21] When we fix one of the particles on the slope and employ the same force law as Eq. (17), then we have the duration of contact for the normal collision without gravity $\tau_{w}=\pi / \sqrt{k_{n}-\eta_{n}^{2} / 4}$ for identical particles. The force law for the flat boundary that replace $2 M$ in Eq. (77) by the mass of the colliding particle $m$ gives the same duration of contact with the above one for the normal collision.

[22] As for the softest case, $1 / k_{n}=2^{7} \times 10^{-5}$, we had to include the data whose energy goes below the lower limit because of the general tendency that the energy becomes small for the steady part of the very soft region. For $1 / k_{n} \lesssim 10^{-5}$, however, we did not see any general trend in the average energy. 


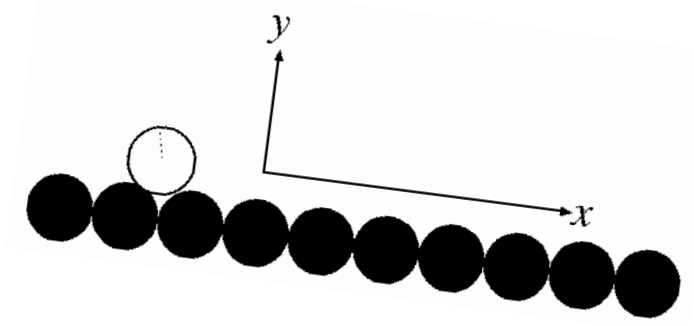

FIG. 1: A snapshot of a single ball rolling down a rough slope.

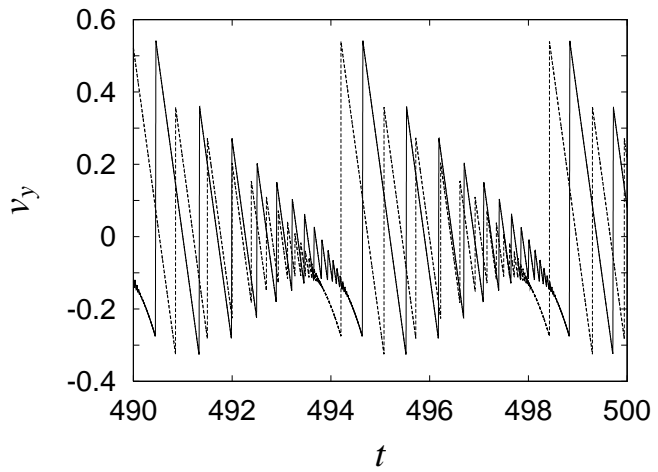

FIG. 2: Time evolution of $v_{y}$ with $k_{n}=2^{-1} \times 10^{5}$ (solid line) and $2^{17} \times 10^{5}$ (dashed line). 

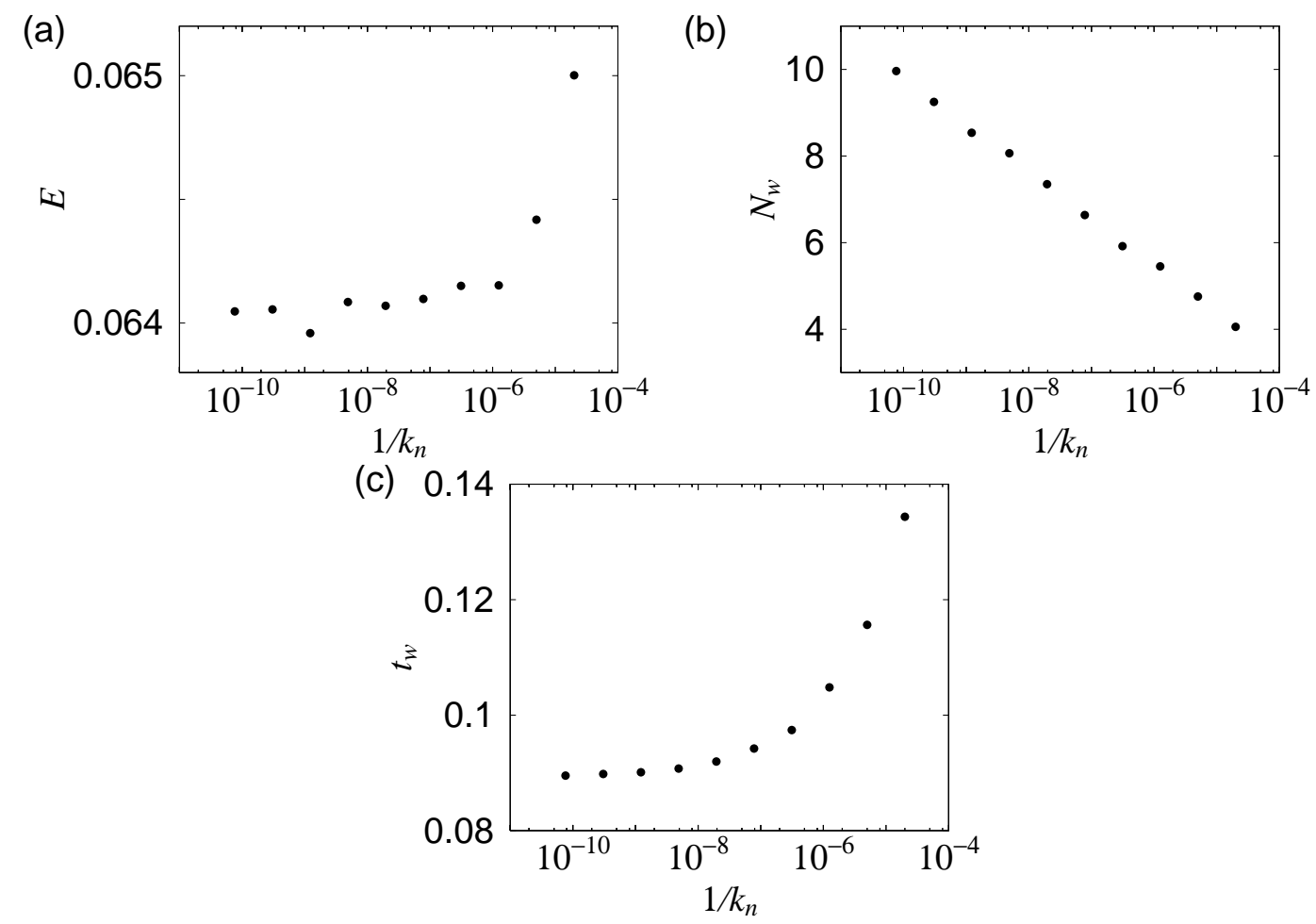

FIG. 3: The stiffness dependence of (a) the time averaged kinetic energy of the particle $E$, (b) the collision rate between the slope and the particle $N_{w}$, and (c) the contact time fraction between the slope and the particle $t_{w}$.

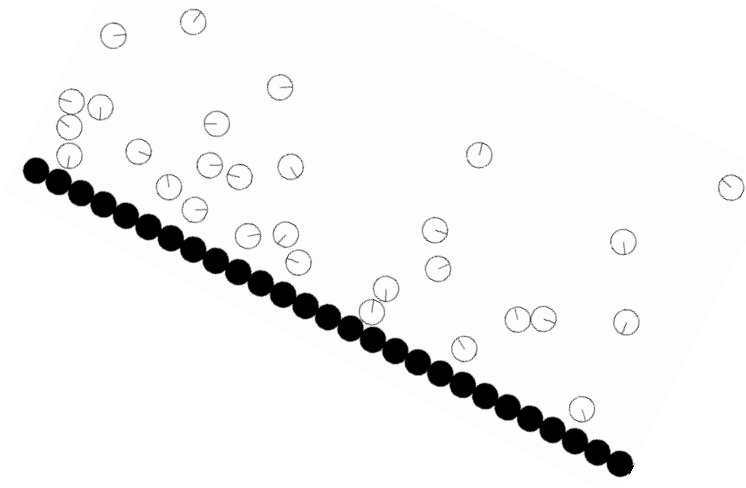

FIG. 4: A snapshot of the dilute collisional flow. 
(a)
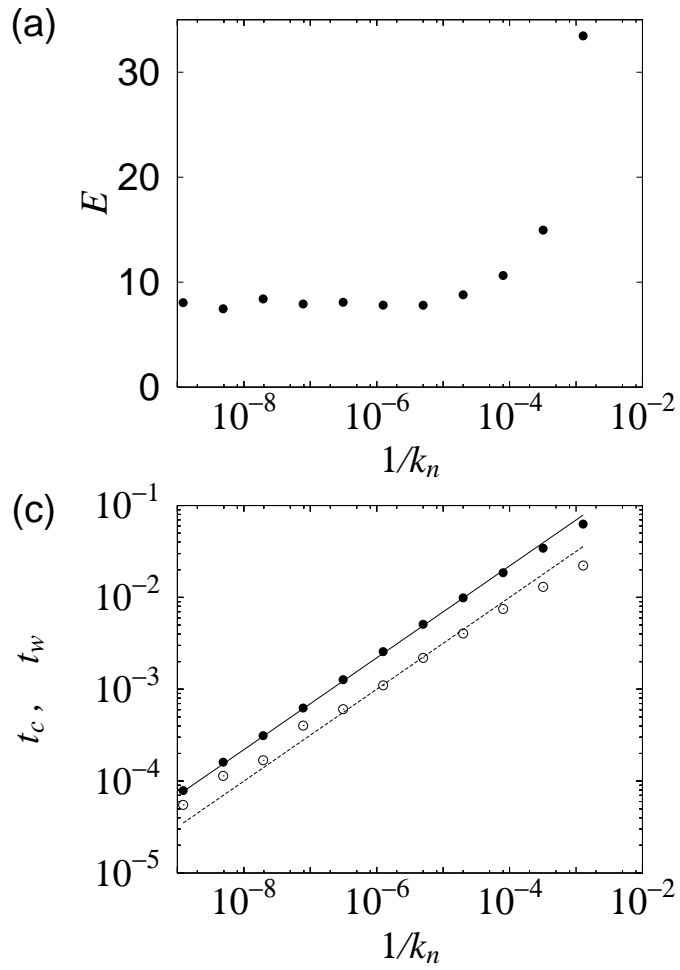

(b)

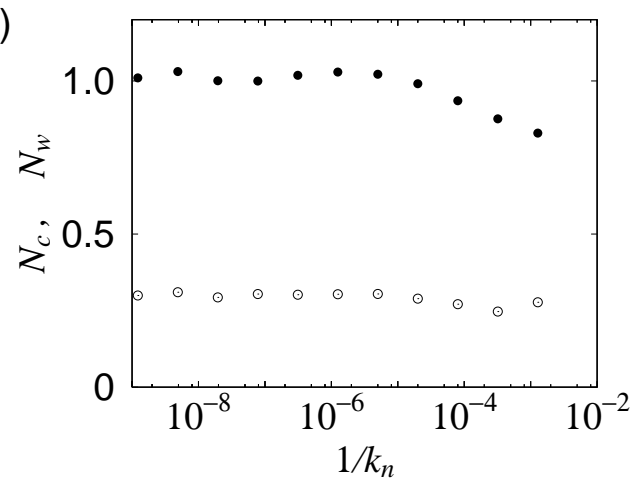

(d)

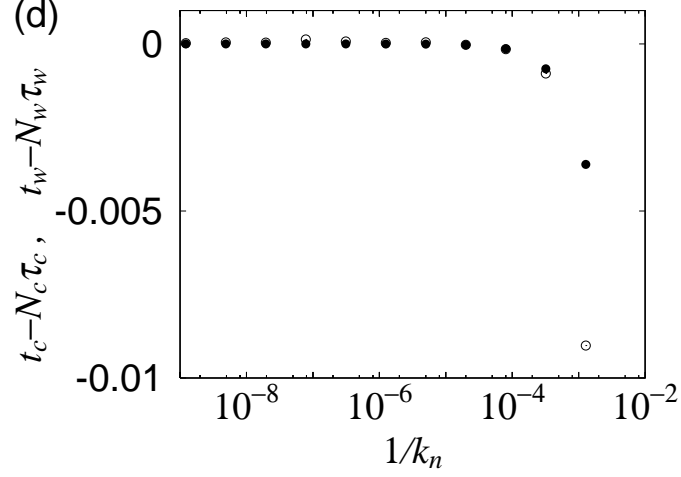

FIG. 5: The stiffness dependences of (a) the averaged kinetic energy per one particle $E$, (b) the averaged collision rates between particles $N_{c}$ (filled circles) and between particles and the floor $N_{w}$ (open circles), (c) the averaged contact time fractions between particles $t_{c}$ (filled circles) and between particles and the floor $t_{w}$ (open circles), (d) the estimated multiple contact time fractions, $t_{c}-N_{c} \tau_{c}$ (filled circles) and $t_{w}-N_{w} \tau_{w}$ (open circles). The solid and the dashed lines in (c) are proportional to $1 / \sqrt{k_{n}}$.
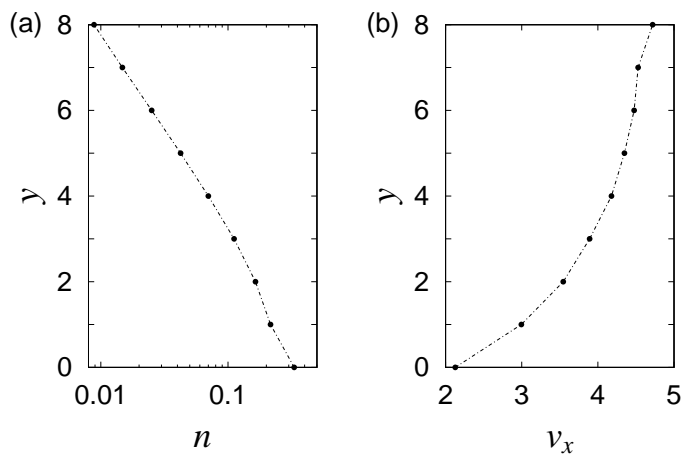

FIG. 6: The number density profile (a) and the velocity profile (b) of the collisional flow. 


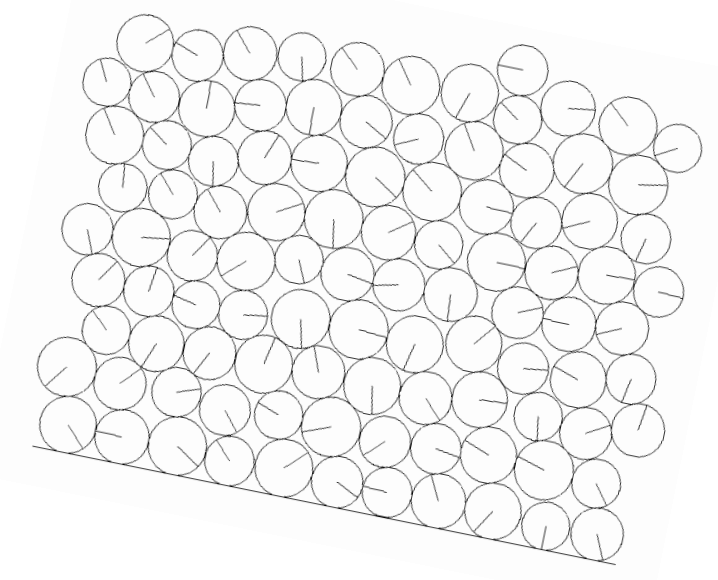

FIG. 7: A snapshot of the dense frictional flow.

(a)

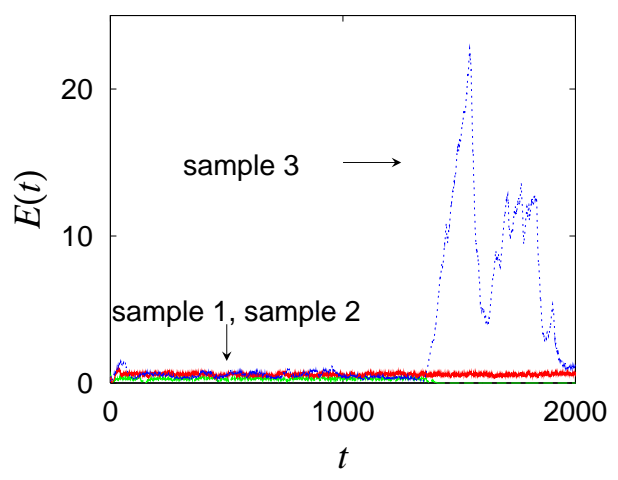

(b)

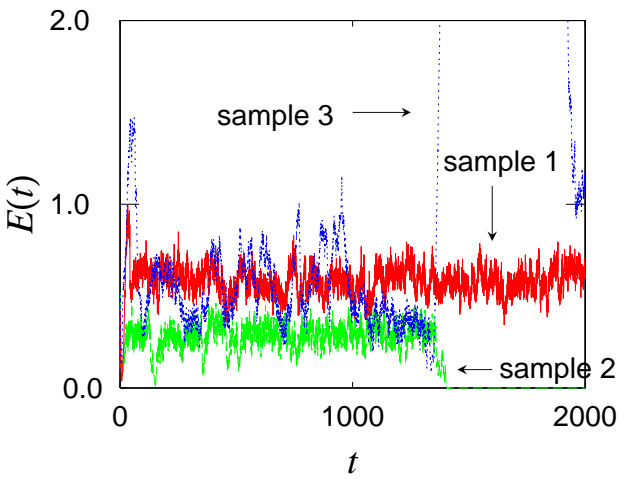

FIG. 8: Time evolution of the kinetic energy per particle of three samples (sample 1: red solid line, sample 2: green dashed line, sample 3: blue dotted line). (b) is the magnification of (a). Sample 1 with $k_{n}=2^{-1} \times 10^{5}$ shows steady behavior within the threshold. Sample 2 and 3 are with $k_{n}=2^{-7} \times 10^{5}$. Sample 2 shows steady behavior for a while but finally stops. $E(t)$ of sample 3 shoots up suddenly at $t \sim 1300$ when one of the particles in the bottom layer runs on other particles. 

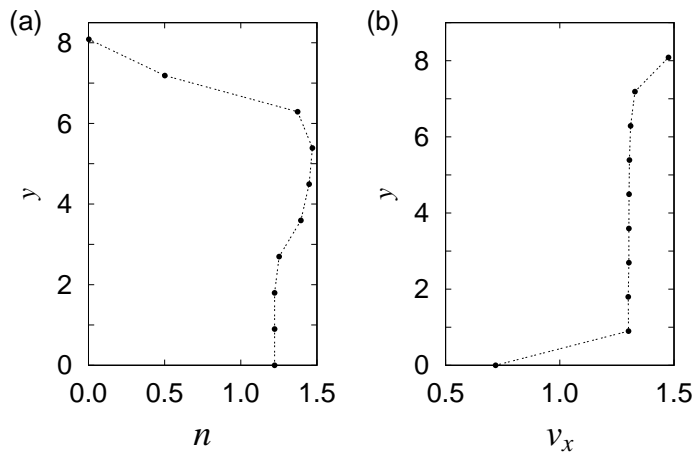

FIG. 9: The density (a) and velocity (b) profiles of the frictional flow.

(a)

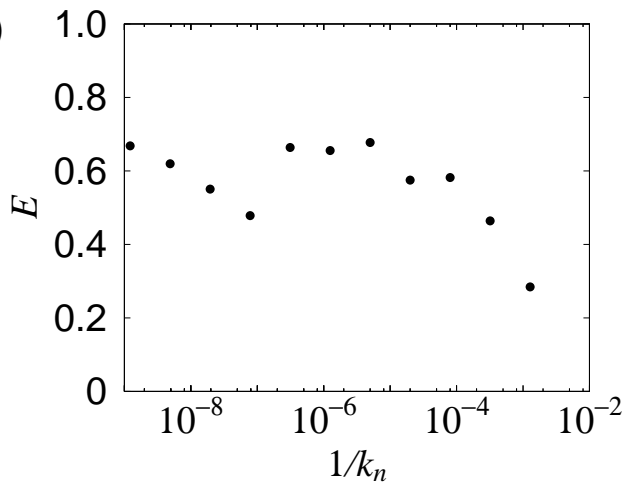

(c)

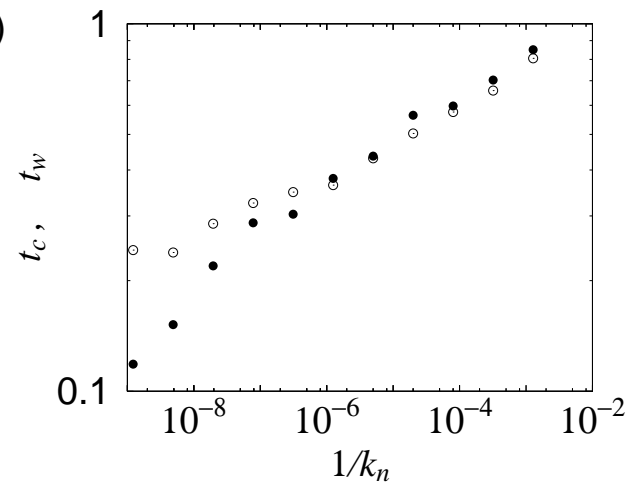

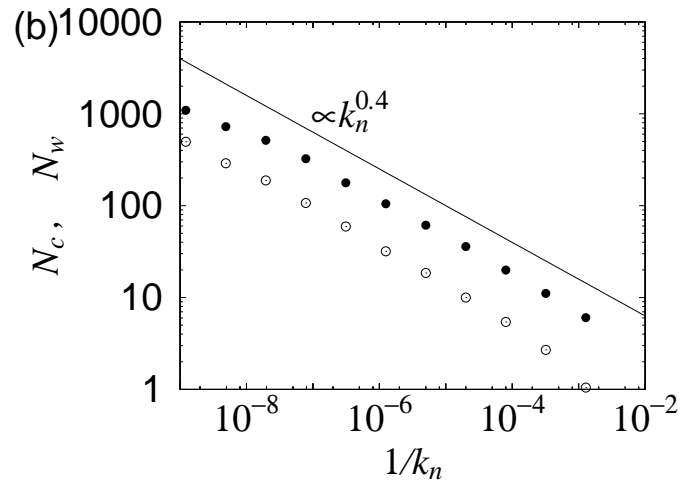

(d)

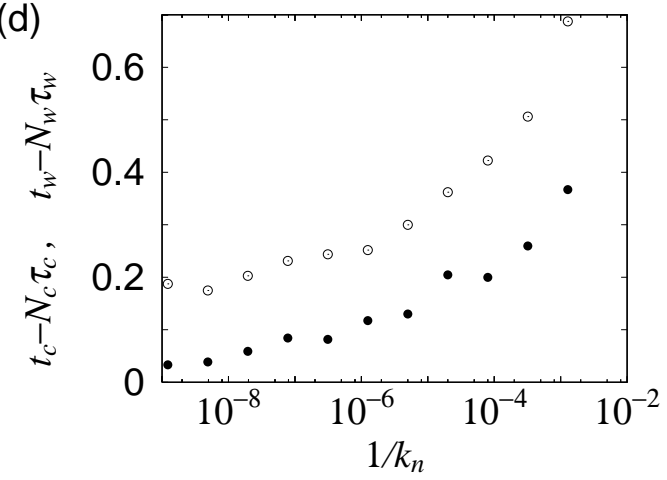

FIG. 10: The stiffness dependences of (a) the averaged kinetic energy $E$, (b) the averaged collision rates between particles $N_{c}$ (filled circles) and between particles and the floor $N_{w}$ (open circles), (c) the averaged contact time fractions between particles $t_{c}$ (filled circles) and between particles and the floor $t_{w}$ (open circles), and (d) the estimated multiple contact time fractions, $t_{c}-N_{c} \tau_{c}$ (filled circles) and $t_{w}-N_{w} \tau_{w}$ (open circles). In (b) the solid line proportional to $k_{n}^{0.4}$ is shown for the guide of the eyes. 